\title{
Pregabalin inhibits excitatory transmission in the central amygdala of the mice with inflammatory pain
}

\author{
Sumii Yamamoto, Yukari Takahashi, Fusao Kato \\ Dept Anesthesiol, Fac Med, Univ Tsukuba, Tsukuba 305-8575, Japan \\ Dept Neurosci, Jikei Univ Sch Med, Tokyo 105-8461, Japan
}

\section{Introduction}

Pregabalin (PGB) is used to treat patients with peripheral and central neuropathic pain in USA, Europe and Japan, and with fibromyalgia in USA and Japan. Apart from its selective binding to the $\alpha 2 \delta$ subunit of voltagegated calcium channels, the targets of PGB in exerting analgesic effects remain unidentified. The central nucleus of amygdala $(\mathrm{CeA})$, a brain region playing essential roles in emotional memory formation, is now recognized to be a kernel site for the enhanced nociception-emotion link in the chronic pain. The CeA receives inputs from the basolateral amygdala (BLA), one of the brain sites showing the highest expression of $\alpha 2 \delta$ mRNA and [3H]PGB binding ${ }^{1)}$, carrying somatosensory information from the thalamocortical network. We examined whether PGB affects the excitatory synaptic transmission from the BLA to CeA in the brain slices prepared from mice with formalin-induced inflammatory pain.

Reference: 1) Trends Pharmacol Sci. 2007, 28:151

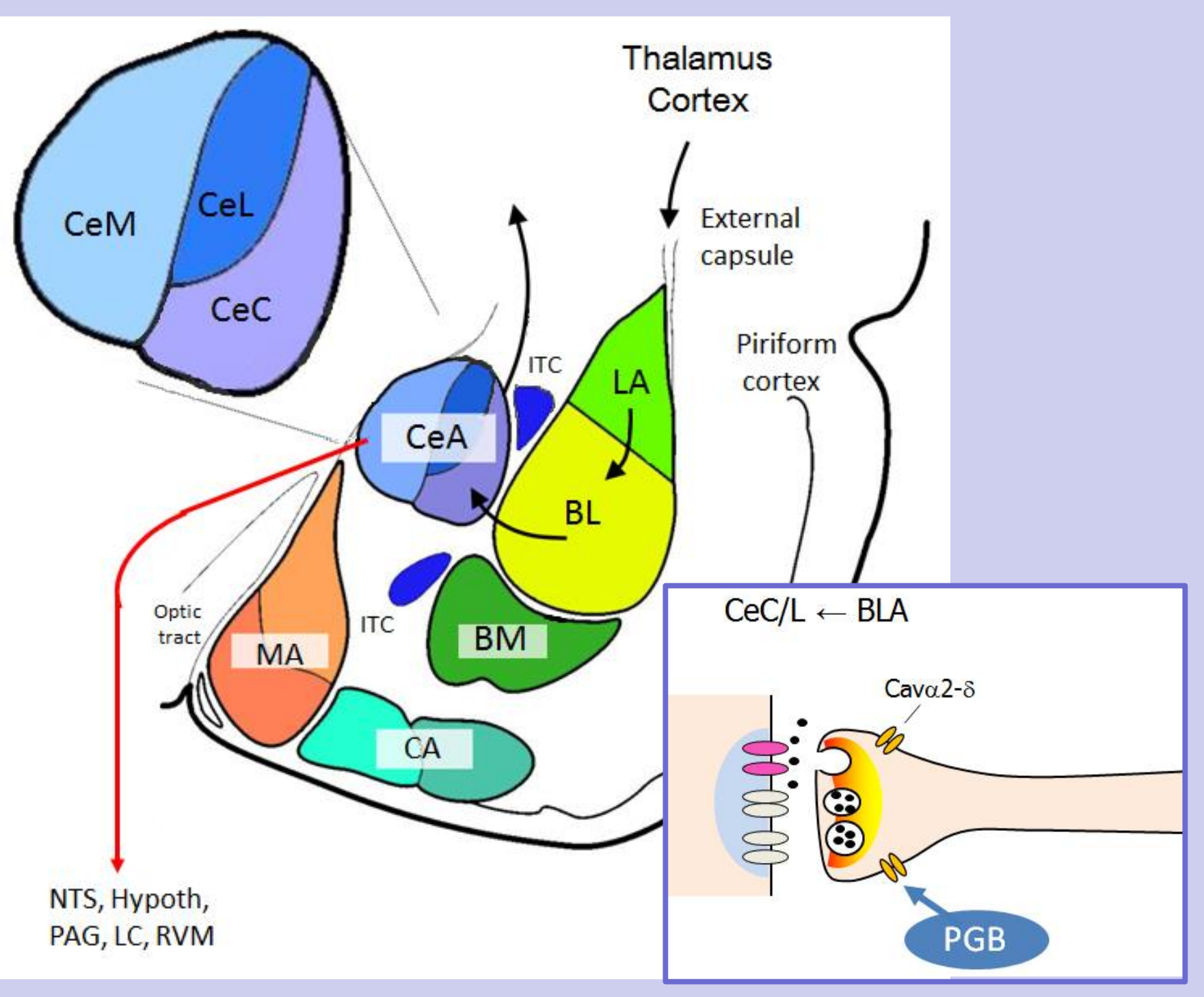

\section{Methods}

C57BL/6 mice (3-8 weeks-old) were used. Inflammation was induced by injecting $20 \mu \mathrm{L}$ of $5 \%$ formalin into the intraplantar surface of the left hind paw. Acute coronal brain slices were prepared 8 hours post-injection. BLAstimulating electrode was placed in the ventral BLA near the borderline to the CeA. Electrically evoked excitatory postsynaptic currents (eEPSCs) were recorded from neurons in the laterocapsular part of the $\mathrm{CeA}(\mathrm{CeL} / \mathrm{C})$ using whole-cell patch-clamp technique. All experiments were carried out at room temperature. PGB was added to external solution and applied in the bath. ttest was used to compare results. All data are presented by the mean \pm SEM.
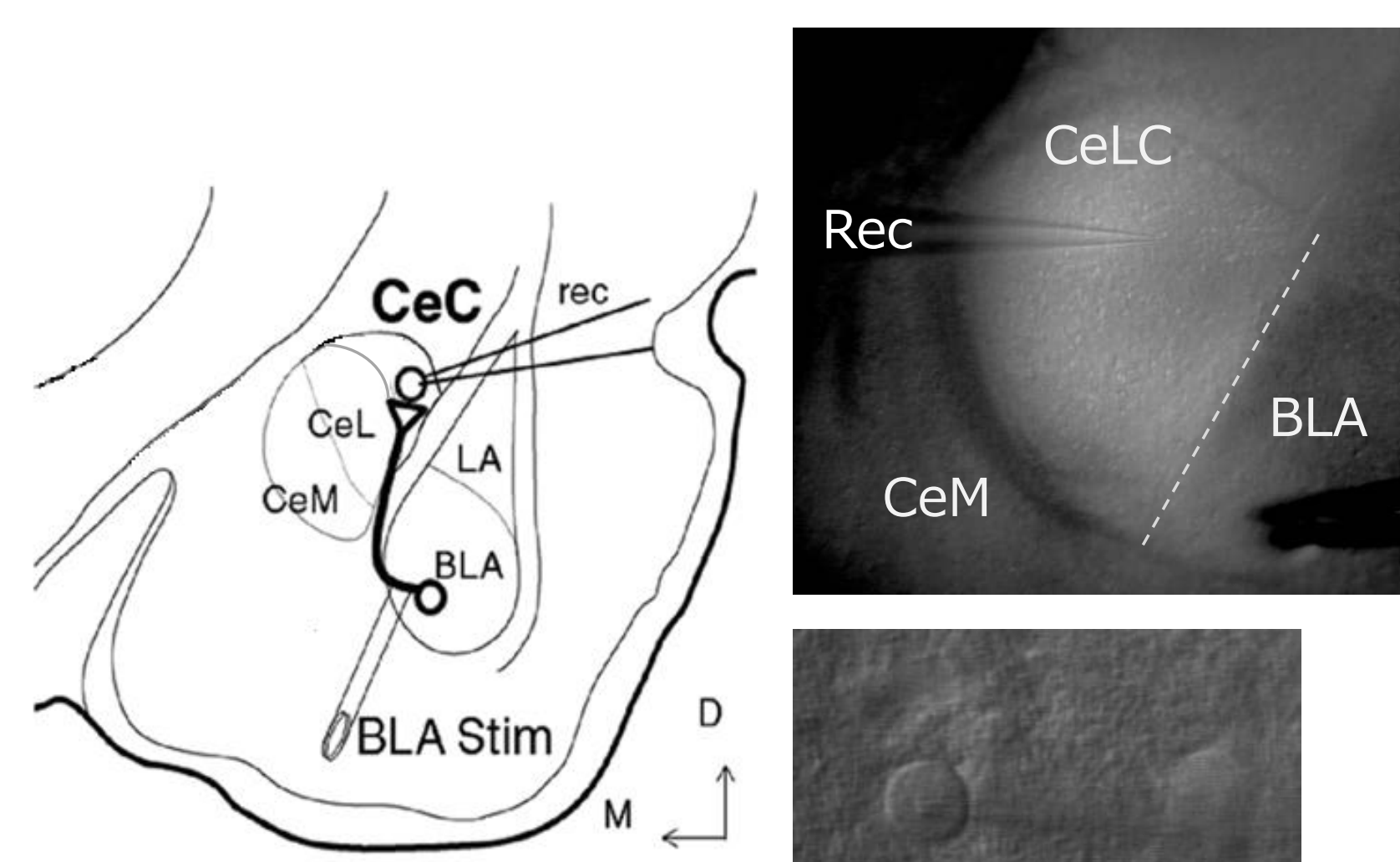

Stim
Results

A PGB $100 \mu \mathrm{M}$

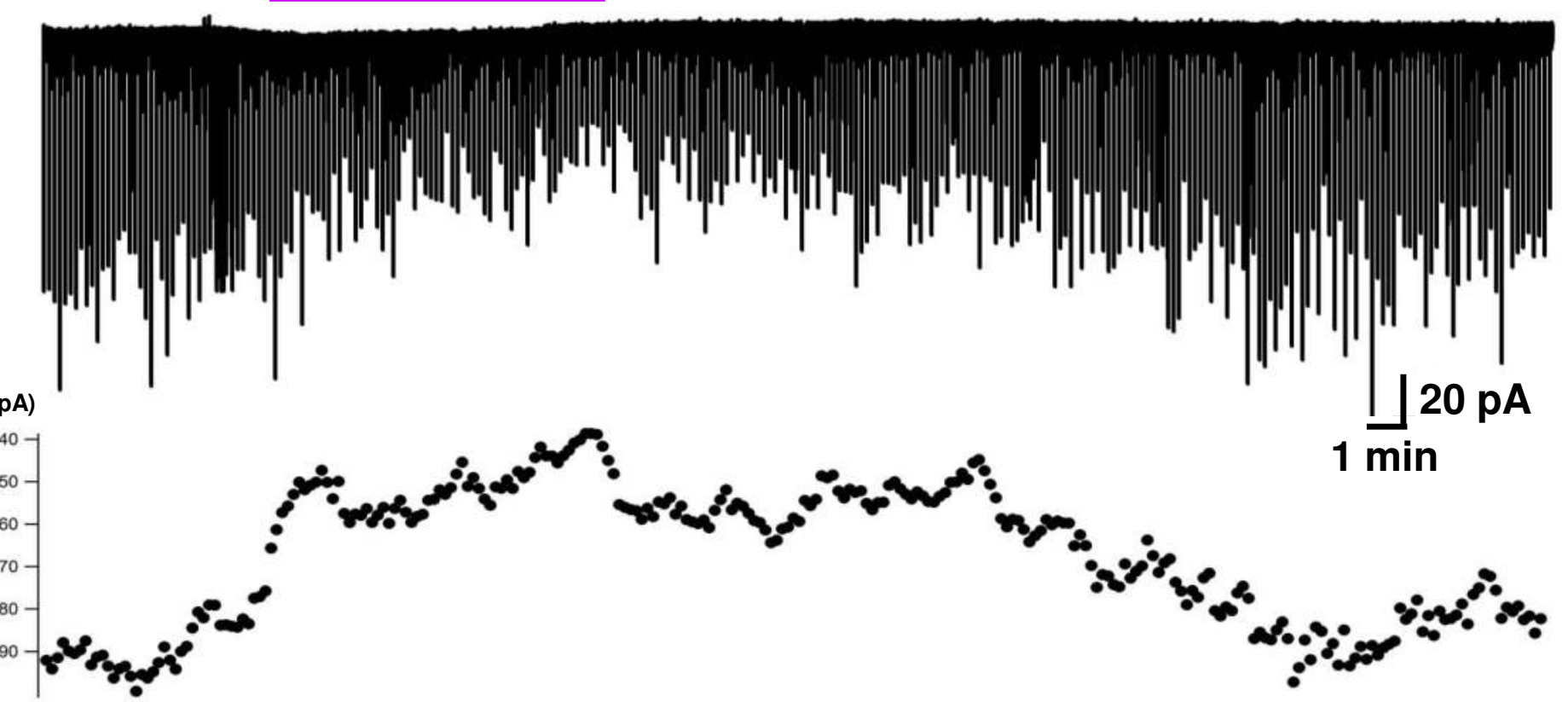

B

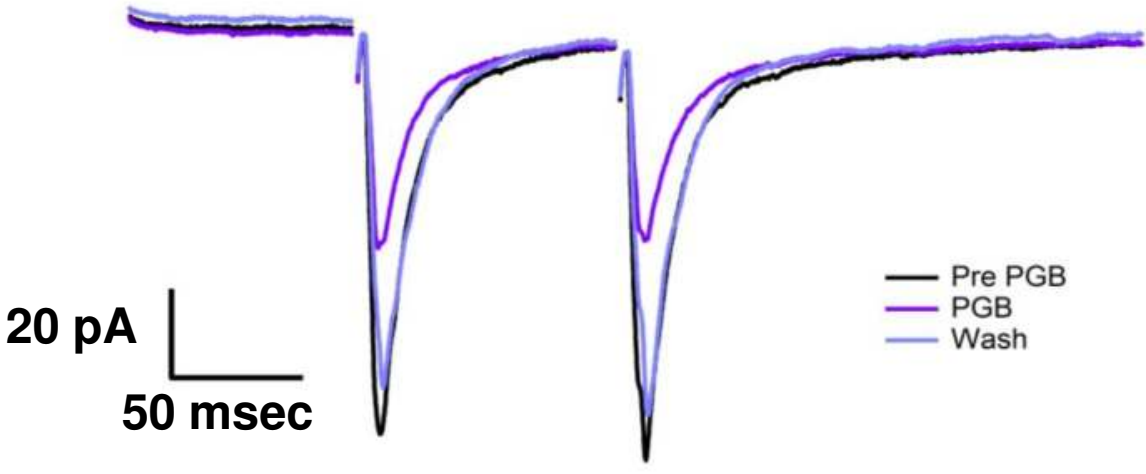

C
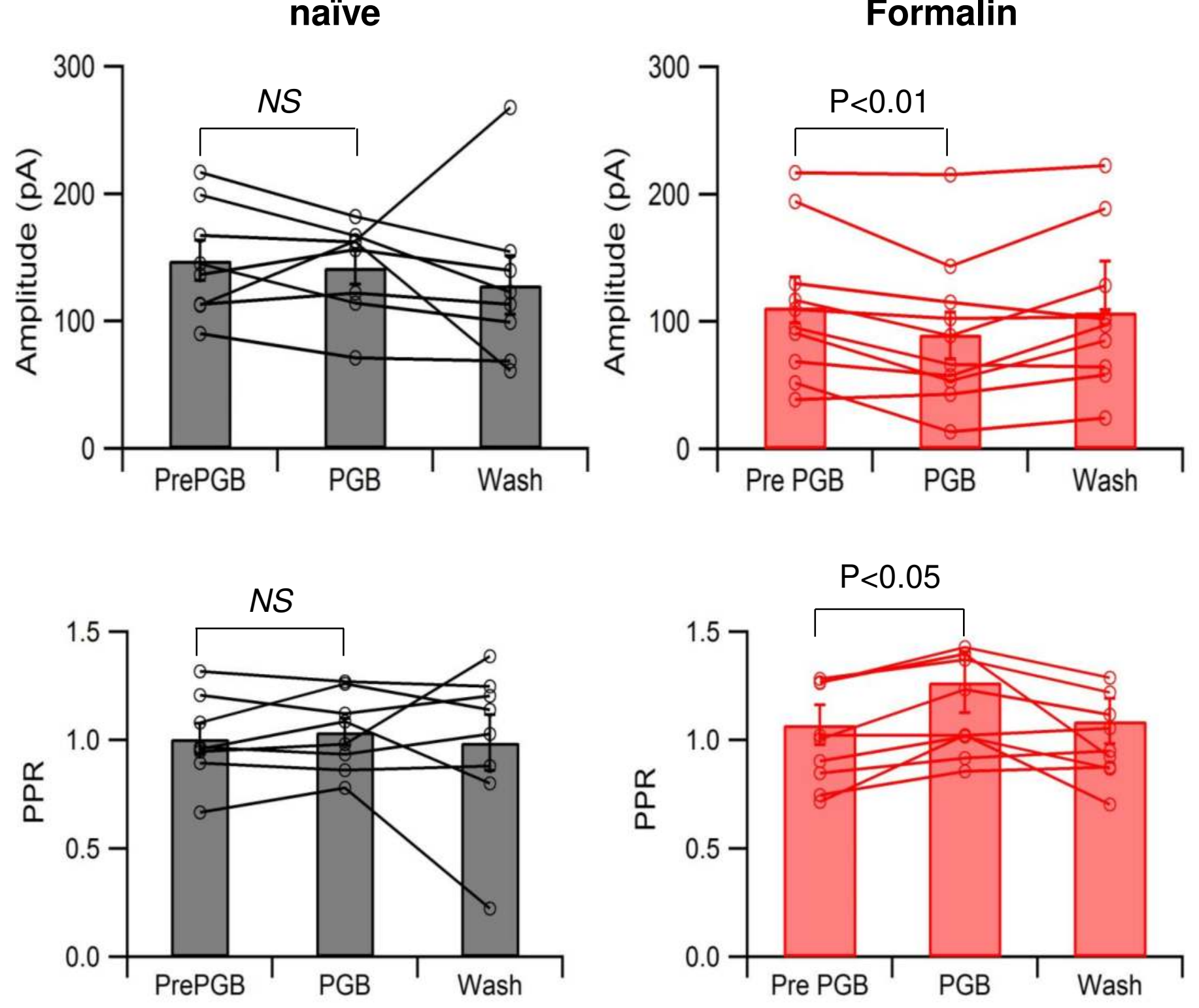

A Typical traces of eEPSCs (top graph) and peak amplitude of first eEPSCs (bottom graph) before, during and after application of PGB $(100 \mu \mathrm{M})$ in the 8 hours after formalin injection.

B Typical traces of eEPSCs before (black), during (purple) and after (blue) application of PGB $(100 \mu \mathrm{M})$ in the 8 hours after formalin injection.

C Summary of the eEPSC amplitude (top) and PPR (bottom) in naïve mice (black, $n=8$ ) and formalin injection mice (red, $n=13$ ). Open circles represent each data from $\mathrm{CeL} / \mathrm{C}$ neurons.

\section{Conclusions}

PGB inhibits excitatory transmission from the BLA to CeA partly through reducing release probability only in inflammation conditions. It is likely that the release machinery in the nerve terminals from the BLA would be a potential target of PGB effect especially in the situation where the inflammatory pain elevates excitatory level of the amygdala network.

This work was supported by JSPS KAKENHI Grant Number 15K08665 Conflict of Interest: No potential COI to disclose 